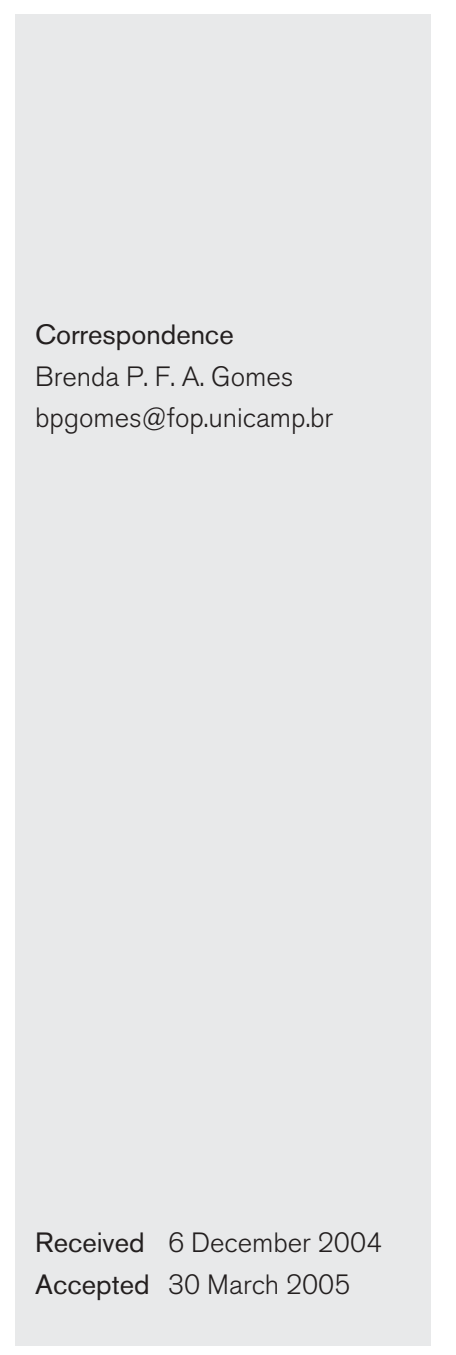

\title{
Quantification of endotoxins in necrotic root canals from symptomatic and asymptomatic teeth
}

\author{
Rogerio C. Jacinto, ${ }^{1,2}$ Brenda P. F. A. Gomes, ${ }^{1}+$ Haroun N. Shah, ${ }^{2} \neq$ \\ Caio C. Ferraz, ${ }^{1}+$ Alexandre A. Zaia ${ }^{1}+$ and Francisco J. Souza-Filho ${ }^{1} \dagger$ \\ ${ }^{1}$ Endodontic Department, Piracicaba Dental School, State University of Campinas, UNICAMP, \\ Avenida Limeira 901, Piracicaba 13414-018, Brazil \\ ${ }^{2}$ NCTC - Molecular Identification Service Unit, Centre for Infections, Health Protection Agency, \\ London NW9 5HT, UK
}

\begin{abstract}
The purpose of this investigation was to quantify the concentration of endotoxin in necrotic root canals and investigate the possible relationship between the concentration of endotoxin and endodontic signs and symptoms. Samples were collected from root canals of 50 patients requiring endodontic treatment due to necrosis of the pulpal tissue. Anaerobic techniques were used to determine the number of c.f.u. in each sample. A quantitative chromogenic Limulus amoebocyte lysate assay was used to measure the concentration of endotoxin in each sample. The presence of c.f.u. was detected by culture in all samples (range $\left.10^{2}-5 \times 10^{6}\right)$. In samples from cases of patients with spontaneous pain, the mean c.f.u. was $1.43 \times 10^{6}$ while in asymptomatic cases it was $9 \cdot 1 \times 10^{4}$. Endotoxin was present in all the samples studied [range 2390.0-22100.0 endotoxin units $(E U) \mathrm{ml}^{-1}$ ]. The mean concentration of endotoxin in samples from patients with spontaneous pain was $18540 \cdot 0 \mathrm{EU} \mathrm{ml}^{-1}$ while in asymptomatic cases it was $12030 \cdot 0 \mathrm{EU} \mathrm{ml}^{-1}$. Asymptomatic cases generally had lower levels of endotoxin (i.e. a negative association). A positive association was found between endotoxin and symptomatic cases (e.g. spontaneous pain, tenderness to percussion, pain on palpation, swelling and purulent exudates). This study showed that endotoxin is present in high concentrations in root canals of symptomatic teeth. There was a positive correlation between the concentration of endotoxin in the root canal and the presence of endodontic signs and symptoms.
\end{abstract}

\section{INTRODUCTION}

Infection of the dental pulpal tissue, which normally progresses to apical periodontitis, is mostly caused by mixed bacterial flora (Baumgartner, 1991; Gomes et al., 2004; Rolph et al., 2001). Several studies have indicated that there is a relationship between polymicrobial infection of root canals, especially Gram-negative anaerobic species, and clinical signs and symptoms such as spontaneous pain, tenderness to percussion, pain on palpation, swelling and purulent exudates (Gomes et al., 1994, 1996a, b, 2004; Griffee et al., 1980; Haapasalo et al., 1986; Hashioka et al., 1992; Jacinto et al., 2003; Morrison \& Kline, 1977).

Gram-negative bacteria contain, in the outermost membrane

†Present address: Endodontic Department, Piracicaba Dental School, State University of Campinas, UNICAMP, Avenida Limeira 901, Piracicaba 13414-018, Brazil.

¥Present address: NCTC - Molecular Identification Service Unit, Centre for Infections, Health Protection Agency, London NW9 5HT, UK.

Abbreviation: LAL, Limulus amoebocyte lysate. of their cell wall, an LPS complex generally referred to as endotoxin that is capable of inducing many biological effects (e.g. complement activation, cytotoxicity and bone resorption). The polysaccharide moiety is a potent antigen that can elicit antibody formation even in submicrogram concentrations (Elin \& Wolff, 1976). Endotoxins may also evoke pain through activation of the Hageman factor or through neurotoxic properties when acting on presynaptic nerve terminals (Seltzer \& Farber, 1994).

Some investigations have shown that the root canal system can act as a pathway for release of microbes and other potential antigens into the periapical tissues (Dahlen, 1980; Matsushita et al., 1999; Pitts et al., 1982; Rosengren \& Winblad, 1975). Anaerobic Gram-negative bacteria have been frequently isolated from root canals of endodontically involved teeth; consequently, their endotoxins may affect the periapical tissues and exert a role in the pathogenesis of inflammatory lesions of pulpal origin (Dahlen \& Hofstad, 1977).

The presence of endotoxin has been reported in samples taken from necrotic pulp (Dahlen \& Bergenholtz, 1980) and 
from the pulpal dentinal walls of periapically involved teeth (Horiba et al., 1990). Correlations have been found between the endotoxin content of infected root canals and clinical endodontic symptoms such as spontaneous pain, tenderness to percussion, exudation and periapical radiolucent areas (Horiba et al., 1991; Schein \& Schilder, 1975). However, few studies have used sensitive methods to quantify the endotoxin content in root canals with necrotic pulpal tissue. Therefore, the purpose of this investigation was to quantify the concentration of endotoxin in necrotic root canals and investigate the possible relationship between the concentration of endotoxin and endodontic signs and symptoms.

\section{METHODS}

Patient selection. Fifty patients who attended the Dental School of Piracicaba, Sao Paulo, Brazil, needing endodontic treatment were included in this research as long as they presented necrotic pulp tissues and showed radiographic evidence of apical periodontitis. Samples were collected from 50 root canals. A detailed medical and dental history was obtained from each patient. Patients who had received antibiotic treatment during the last 3 months or who had a general disease were excluded from the study. The teeth used in this study were, mostly, nonintact; those that could not be fully isolated with a rubber dam were excluded. The Human Volunteers Research and Ethics Committee of the Dental School of Piracicaba approved a protocol describing the specimen collection for this investigation, and all patients signed an informed consent document to participate in the study.

Clinical features. The following characteristics were recorded for each patient so that they could be correlated with the findings: age, gender and tooth type. Clinical symptoms and signs recorded included: nature of pain, history of previous pain, tenderness to percussion, pain on palpation, mobility, presence of a sinus and its origin (endodontic or periodontal), presence of swelling of the periodontal tissues, probing depth of the periodontal pocket, history of previous and present antibiotic therapy and any other relevant medication, radiographic findings and the internal status of the canal (such as dry canal or the presence of clear, haemorrhagic or purulent exudates, which were detected as a distinct dampening or stain on the sampling paper points). Each type of exudate was analysed alone and also grouped with the other types under the denomination 'wet canal'. Patients with negative responses to all the symptoms were considered asymptomatic. Patients with positive responses to one or more of the following symptoms were considered symptomatic: spontaneous pain, pain on palpation, tenderness to percussion, swelling and purulent exudates.

Sampling procedure. The method followed for the microbiological procedures has been described previously (Gomes et al., 1994, 2004; Jacinto et al., 2003).

Samples were collected using strict asepsis. In multi-rooted teeth only the largest root canal was sampled, in order to confine the microbial evaluation to a single ecological environment. A rubber dam was used to isolate the tooth. The tooth and the surrounding field were then cleansed with $30 \% \mathrm{v} / \mathrm{v}$ hydrogen peroxide and decontaminated with a $2.5 \% \mathrm{v} / \mathrm{v}$ sodium hypochlorite solution for $30 \mathrm{~s}$ each. The solution was inactivated with sterile $5 \% \mathrm{v} / \mathrm{v}$ sodium thiosulfate. Access to the root canal was made using sterile burs without water spray. A sterile pyrogenfree paper point (size 35; Dentsply-Maillefer) was then introduced in the full-length of the canal and retained in position for $60 \mathrm{~s}$ for sampling. Root canals in which the paper point could not be introduced to its full length were not included in the research. If the root canal was dry, $5 \mu \mathrm{l}$ of sterile pyrogen-free saline solution was introduced into the canal to ensure viable sample acquisition. Chemical active irrigants were never used before sampling.

The paper point was inserted in the canal to the approximate canal length determined radiographically. The procedure was repeated with five paper points. A sterilized pyrogen-free saline solution $(1 \mathrm{ml})$ was used to transport the samples to the laboratory, where aliquots of $100 \mu \mathrm{l}$ were used for microbial cultivation and the rest of the sample was frozen at $-30{ }^{\circ} \mathrm{C}$, as recommended by the manufacturer, until the next step of the procedure.

Microbial cultivation. The $100 \mu \mathrm{l}$ aliquots of the samples were used for serial 10-fold dilutions up to $10^{-4}$ in tubes containing Fastidious Anaerobe Broth (FAB, Lab M, Bury, UK). Fifty microlitres of the serial dilutions were plated, using sterile plastic spreaders, into $5 \%$ defibrinated sheep blood Fastidious Anaerobe Agar (FAA, Lab M) to culture non-selectively obligate anaerobes and facultative anaerobes. The plates were incubated at $37^{\circ} \mathrm{C}$ in an anaerobic atmosphere for up to 14 days. After this period, the c.f.u. were visually quantified from each plate.

Quantitative chromogenic Limulus amoebocyte lysate assay. During the procedures a series of precautions were taken to avoid contamination of the samples. All materials coming into contact with samples were endotoxin-free. Careful techniques were used to avoid microbial or endotoxin contamination. Strict adherence to the time and temperature specified in the manufacturer's instructions was maintained throughout the test procedure.

The endotoxin standard curve for the quantitative chromogenic Limulus amoebocyte lysate (LAL) assay (QCL-1000; BioWhitaker) was generated following the manufacturer's procedure. It was plotted as a parameter for calculation of the concentration of endotoxin in the sample using the endotoxins supplied in the kit (Escherichia coli O111:B4) with a known concentration [23 endotoxin units (EU) $\mathrm{ml}^{-1}$ ]. After the LAL incubation, the absorbances of endotoxin standard solutions at a series of endotoxin concentrations (i.e. 0·05, 0·1, 0.25 and $\left.0.5 \mathrm{EU} \mathrm{ml} \mathrm{m}^{-1}\right)$ were measured individually using an ELISA reader (Ultramark, Bio-Rad). Samples were run in duplicate using endotoxin-free water as the blank. Standard endotoxin curves were obtained by plotting each absorbance against the corresponding concentration. Four standard endotoxin solutions were prepared with concentrations of $0.05,0 \cdot 1,0.25$ and $0.5 \mathrm{EU} \mathrm{ml}^{-1}$. The absorbance values of the endotoxin solutions previously prepared were spectrophotometrically measured at $405 \mathrm{~nm}$ in the ELISA reader. The absorbance at $405 \mathrm{~nm}$ was linear within the concentration range used. The linearity of the standard curve within the concentration range used to predict endotoxin values was verified based on the least-squares value. A best-fit straight line among these points was drawn and the least-squares value $\left(\mathrm{R}^{2}\right)$ was obtained directly (Microsoft Excel). The reproducibility can be verified by comparing the different curves.

Test procedure. Serial dilutions of the samples were made to $10^{-4}$. LAL reagent water (blank) was used as a negative control. All reactions were accomplished in duplicate to validate the test. A 96-well microplate (Corning Costar) was used in a heating block at $37^{\circ} \mathrm{C}$, and maintained throughout the assay. Initially, $50 \mu \mathrm{l}$ of the blank was added, followed by the standard endotoxin solutions and the samples consecutively added to the wells. This was followed by the addition of $50 \mu \mathrm{LAL}$ to each well using a multi-channel pipette and reagent reservoir, and then the microplate was briefly mixed. Ten minutes later $100 \mu$ of substrate solution (pre-warmed to $37^{\circ} \mathrm{C}$ ) was added to each well, maintaining always the same sequence. The plate was mixed and incubated at $37^{\circ} \mathrm{C}$ for $6 \mathrm{~min}$, after which $100 \mu \mathrm{l}$ of a stop reagent (acetic acid, $25 \% \mathrm{v} / \mathrm{v}$ ) was added to each well. The absorbance $(405 \mathrm{~nm})$ was read using a spectrophotometer (Ultrask, Bio-Rad) 
Calculation of endotoxin concentration. The mean absorbance value of the blank was subtracted from the mean absorbance value of the standards and the value of samples to calculate the mean absorbance of the samples. Since this absorbance value was directly proportional to the concentration of endotoxin present, the endotoxin concentration was determined from the standard curve.

Statistical analysis. The data collected were statistically analysed using Intercooled Stata 8.2 for Windows (StataCorp). Either a Pearson Chi-squared test or a one-sided Fisher's exact test, as appropriate, was chosen to test the null hypothesis that there was no relationship between the concentration of endotoxin present in the root canal and the clinical endodontic signs and symptoms. Additionally, locally weighted regression plots were drawn of signs and symptoms against number of c.f.u. or concentration of endotoxin. The LOESS (locally weighted regression, also known as LOWESS) method of smoothing was used. This method of regression is useful in situations in which the classical linear regression procedures do not perfom well; here this is because much of the data is binary (i.e. either 0 or 1). LOESS is based on the ideas that any function can be well approximated by low-order local polynomials and that high-degree polynomials would tend to overfit the data. One disadvantage of LOESS is that it does not produce a regression function that is easily represented by a mathematical formula; another is that it is computationally intensive. A user-specified input (the bandwidth) determines how much of the data is used to fit each local polynomial; the value used was that automatically set by Stata.

\section{RESULTS AND DISCUSSION}

The presence of c.f.u. was detected by culture in all samples (range $10^{2}-5 \times 10^{6}$ ). In the samples from symptomatic cases the median of c.f.u. was $8.7 \times 10^{5}$ while in asymptomatic cases the median was $5 \times 10^{3}$. The confidence interval for the means of c.f.u. in symptomatic cases was $6.8 \times 10^{5}-$ $1.7 \times 10^{6}$ around a mean of $1.7 \times 10^{6}$ while in asymptomatic cases the interval was $1.8 \times 10^{4}-2 \times 10^{5}$ around a mean of $9 \cdot 1 \times 10^{4}$.

Table 1 shows the signs and symptoms, number of c.f.u. and concentration of endotoxin detected for each patient. Logistic regression was used to compare c.f.u. values with the symptoms. When c.f.u. were compared to asymptomatic cases a negative-log association was observed, indicating that as the c.f.u. values increase, the number of asymptomatic cases decreases. The opposite was observed when c.f.u. values were compared to the symptomatic cases. A locally weighted regression plot was drawn to visualize this information (Fig. 1). This shows that there are greater numbers of c.f.u. in symptomatic cases than in asymptomatic cases.

The LAL assay (QCL-1000) indicated that endotoxin was present in all of the samples studied. The range of endotoxin quantified in the samples was $2390 \cdot 0-22100 \cdot 0 \mathrm{EU} \mathrm{ml}^{-1}$. The median concentration of endotoxin in samples from symptomatic cases was $20888.0 \mathrm{EU} \mathrm{ml}^{-1}$ while in asymptomatic cases it was $15145 \cdot 0 \mathrm{EU} \mathrm{ml}^{-1}$. Confidence intervals for the means for symptomatic cases were $16600 \cdot 0-20300 \cdot 0$ around a mean of $18500 \cdot 0$, and for asymptomatic cases were $8300 \cdot 0-$ $15800 \cdot 0$ around a mean of $12000 \cdot 0$. For the continuous values of endotoxin, box and whisker plots were constructed to show the concentration of endotoxin present in both symptomatic and asymptomatic cases (Fig. 2). There was a wider range of concentrations for the asymptomatic cases although in symptomatic cases there was a significant increase in the concentration of endotoxin (Fig. 2).

The association between the concentration of endotoxin and the occurrence of asymptomatic cases is statistically shown in Fig. 3(a). The associations between the concentration of endotoxin when each of the symptoms were present are shown in Fig. 3(b-f). The locally weighted regression line shows a negative association between the concentration of endotoxin and the occurrence of asymptomatic cases, as demonstrated by the negative gradient of the line (Fig. 3a). On the other hand, there is a positive association between endotoxin and spontaneous pain as the gradient of this line is positive (Fig. 3b). A similar profile was observed when the concentration of endotoxin was compared to the following symptoms: tenderness to percussion, pain on palpation, swelling and purulent exudates (Fig. 3c-f).

The present study focused on the relationship between the concentration of endotoxin present in root canals of infected teeth and endodontic signs and symptoms. Samples were collected from symptomatic and from asymptomatic teeth using aseptic techniques and taking particular care to avoid contamination with endotoxins from areas other than the root canal. A chromogenic LAL test (QCL-1000) was used to measure the endotoxin found in the root canals. This method utilizes a modified Limulus amoebocyte lysate and a synthetic colour-producing substrate to detect and quantify chromogenically the endotoxin of Gram-negative bacteria. Recent reports of Khabbaz et al. $(2000,2001)$ have also used this method to quantify endotoxin present in dental caries and inflamed pulp tissues, which proved to be reliable even for identifying low concentrations of endotoxin.

Due to its sensitivity, materials other than endotoxin, such as proteins, nucleic acids and peptidoglycan from Gram-positive bacteria, and compounds found in inflammatory exudates, may interfere with the assay, inducing or giving falsepositive or -negative results. However, dilutions of the test samples in LAL reagent water up to $10^{-4}$ and heating at $70{ }^{\circ} \mathrm{C}$ were used in the present study for the removal of non-specific products as reported by Friberger et al. (1982).

Infected root canals are characterized by polymicrobial infections, and symptomatic infected teeth tend to harbour a larger number of bacteria than asymptomatic ones (Jacinto et al., 2003; Sundqvist et al., 1989). In the present research the mean number of c.f.u. was higher in symptomatic (mean $1.43 \times 10^{6}$ ) than in asymptomatic cases (mean $9 \cdot 1 \times 10^{4}$ ). Several species of bacteria have been isolated from endodontic infections of symptomatic teeth, with a predominance of Gram-negative obligate anaerobes, especially Fusobacterium species and black-pigmented bacteria (Gomes et al., 1994, 1996a, b, 2004; Hashioka et al., 1992; Jacinto et al., 2003; Yoshida et al., 1987).

Endotoxins are potent inflammatory agents that activate the classical and alternative pathways of complement system (Morrison \& Kline, 1977). Complement activation releases 
Table 1. Presence of signs and symptoms, c.f.u. and amount of endotoxin for each patient

Asympt, asymptomatic; PoP, pain on palpation; SP, spontaneous pain; TTP, tenderness to percussion.

\begin{tabular}{|c|c|c|c|c|c|c|c|c|}
\hline \multirow{2}{*}{$\begin{array}{l}\text { Case } \\
\text { no. }\end{array}$} & \multicolumn{6}{|c|}{ Clinical signs and symptoms } & \multirow[t]{2}{*}{ No. of c.f.u. } & \multirow{2}{*}{$\begin{array}{l}\text { Endotoxin } \\
\left(\mathrm{EU} \mathrm{ml} \mathrm{m}^{-1}\right)\end{array}$} \\
\hline & Asympt & SP & TTP & PoP & Swelling & Exudate & & \\
\hline 1 & No & Yes & No & Yes & Yes & Yes & $3 \times 10^{6}$ & $4240 \cdot 0$ \\
\hline 2 & Yes & No & No & No & No & $\mathrm{No}^{*}$ & $3 \cdot 8 \times 10^{3}$ & $21420 \cdot 0$ \\
\hline 3 & No & Yes & No & Yes & Yes & Yes & $5 \times 10^{6}$ & $21970 \cdot 0$ \\
\hline 4 & No & No & No & Yes & Yes & No & $1.5 \times 10^{5}$ & $17430 \cdot 0$ \\
\hline 5 & Yes & No & No & No & No & No & $4 \times 10^{2}$ & $15140 \cdot 0$ \\
\hline 6 & No & Yes & Yes & Yes & Yes & Yes & $1.6 \times 10^{5}$ & $17670 \cdot 0$ \\
\hline 7 & No & Yes & Yes & Yes & Yes & Yes & $8 \times 10^{3}$ & $17210 \cdot 0$ \\
\hline 8 & Yes & No & No & No & No & $\mathrm{No}^{*}$ & $1.2 \times 10^{3}$ & $2430 \cdot 0$ \\
\hline 9 & No & Yes & Yes & Yes & No & No & $2 \times 10^{2}$ & $5620 \cdot 0$ \\
\hline 10 & No & Yes & Yes & Yes & No & No & $1.92 \times 10^{5}$ & $18270 \cdot 0$ \\
\hline 11 & Yes & No & No & No & No & $\mathrm{No}^{*}$ & $9 \cdot 8 \times 10^{4}$ & $20810 \cdot 0$ \\
\hline 12 & No & No & Yes & Yes & No & Yes & $2.3 \times 10^{6}$ & $21660 \cdot 0$ \\
\hline 13 & Yes & No & No & No & No & $\mathrm{No}^{*}$ & $10^{3}$ & $19180 \cdot 0$ \\
\hline 14 & No & No & No & No & Yes & Yes & $6.4 \times 10^{4}$ & $20930 \cdot 0$ \\
\hline 15 & No & Yes & Yes & Yes & Yes & Yes & $2 \times 10^{6}$ & $19260 \cdot 0$ \\
\hline 16 & No & Yes & Yes & Yes & Yes & Yes & $8.3 \times 10^{5}$ & $18990 \cdot 0$ \\
\hline 17 & No & No & No & Yes & No & Yes & $1.4 \times 10^{5}$ & $20890 \cdot 0$ \\
\hline 18 & No & Yes & Yes & Yes & Yes & Yes & $5 \times 10^{5}$ & $21180 \cdot 0$ \\
\hline 19 & No & Yes & Yes & Yes & Yes & No & $1.27 \times 10^{6}$ & $20060 \cdot 0$ \\
\hline 20 & No & Yes & Yes & Yes & Yes & Yes & $1.23 \times 10^{6}$ & $21630 \cdot 0$ \\
\hline 21 & No & Yes & Yes & No & No & No & $8.7 \times 10^{5}$ & $21030 \cdot 0$ \\
\hline 22 & No & No & No & Yes & No & Yes & $1.78 \times 10^{6}$ & $20360 \cdot 0$ \\
\hline 23 & No & No & Yes & No & No & No & $8.9 \times 10^{5}$ & $21480 \cdot 0$ \\
\hline 24 & No & Yes & No & Yes & No & No & $1.34 \times 10^{6}$ & $21140 \cdot 0$ \\
\hline 25 & No & No & No & No & No & No & $1.5 \times 10^{5}$ & $19800 \cdot 0$ \\
\hline 26 & Yes & No & No & No & No & No & $5 \times 10^{4}$ & $5210 \cdot 0$ \\
\hline 27 & No & Yes & Yes & Yes & Yes & Yes & $5 \times 10^{6}$ & $21030 \cdot 0$ \\
\hline 28 & Yes & No & No & No & No & No & $2 \times 10^{2}$ & $4800 \cdot 0$ \\
\hline 29 & No & No & Yes & No & No & No & $1 \cdot 2 \times 10^{4}$ & $18120 \cdot 0$ \\
\hline 30 & Yes & No & No & No & No & $\mathrm{No}^{*}$ & $10^{2}$ & $2390 \cdot 0$ \\
\hline 31 & Yes & No & No & No & No & No & $5 \cdot 4 \times 10^{5}$ & $19190 \cdot 0$ \\
\hline 32 & No & Yes & Yes & Yes & No & No & $1.3 \times 10^{6}$ & $22100 \cdot 0$ \\
\hline 33 & No & Yes & Yes & Yes & Yes & No & $2.7 \times 10^{4}$ & $12770 \cdot 0$ \\
\hline 34 & No & Yes & Yes & Yes & No & Yes & $1.1 \times 10^{5}$ & $21100 \cdot 0$ \\
\hline 35 & No & No & Yes & No & No & No & $2 \times 10^{4}$ & $3500 \cdot 0$ \\
\hline 36 & Yes & No & No & No & No & No & $6 \cdot 2 \times 10^{3}$ & $20240 \cdot 0$ \\
\hline 37 & No & Yes & Yes & Yes & No & Yes & $10^{6}$ & $17720 \cdot 0$ \\
\hline 38 & Yes & No & No & No & No & No & $9 \times 10^{3}$ & $17310 \cdot 0$ \\
\hline 39 & Yes & No & No & Yes & No & No & $2 \times 10^{2}$ & $2390 \cdot 0$ \\
\hline 40 & Yes & No & No & No & No & Yes & $5 \times 10^{3}$ & $16240 \cdot 0$ \\
\hline 41 & Yes & No & No & No & No & No & $3.5 \times 10^{4}$ & $18560 \cdot 0$ \\
\hline 42 & Yes & No & No & No & No & No & $9 \times 10^{4}$ & $5420 \cdot 0$ \\
\hline 43 & Yes & No & No & No & No & $\mathrm{No}^{*}$ & $10^{4}$ & $10330 \cdot 0$ \\
\hline 44 & No & Yes & Yes & No & No & Yes & $1.5 \times 10^{6}$ & $21520 \cdot 0$ \\
\hline 45 & No & Yes & Yes & No & No & Yes & $3 \times 10^{6}$ & $20960 \cdot 0$ \\
\hline 46 & Yes & No & No & No & No & No & $3 \times 10^{2}$ & $2890 \cdot 0$ \\
\hline 47 & Yes & No & No & No & No & No & $8 \cdot 8 \times 10^{5}$ & $20580 \cdot 0$ \\
\hline 48 & No & Yes & No & Yes & No & No & $5 \times 10^{5}$ & $21210 \cdot 0$ \\
\hline 49 & No & Yes & Yes & Yes & Yes & Yes & $2.55 \times 10^{6}$ & $21140 \cdot 0$ \\
\hline 50 & Yes & No & No & No & No & $\mathrm{No}^{*}$ & $10^{3}$ & $4080 \cdot 0$ \\
\hline
\end{tabular}

${ }^{\star}$ Dry canals. 


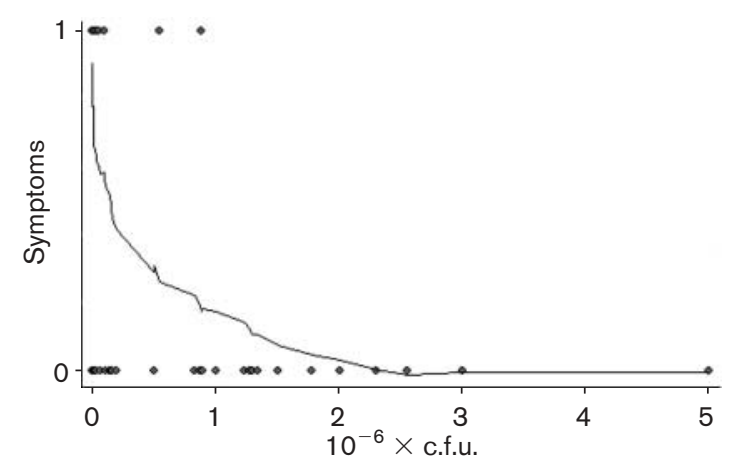

Fig. 1. Locally weighted regression plot of c.f.u. in relation to asymptomatic $(n=19)$ and symptomatic $(n=31)$ cases. Each patient's values are plotted on the graph. Note that 0 on the vertical axis indicates presence of symptoms (i.e. symptomatic cases) and 1 indicates their absence (i.e. asymptomatic cases).

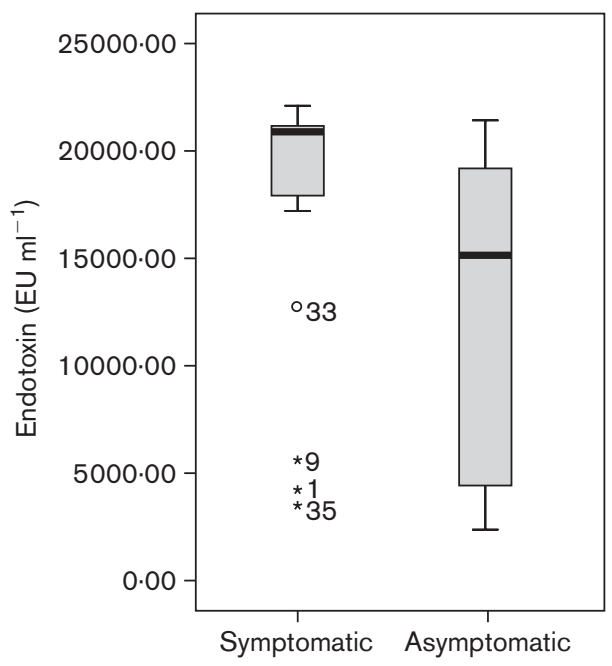

Fig. 2. Box and whisker plots of the concentration of endotoxin in both symptomatic and asymptomatic cases. Symptomatic outliers are indicated by the circle and asterisks, along with the case numbers of these outliers. The number of symptomatic cases was 31, the number of asymptomatic cases was 19 .

biologically active peptides, which mediate a number of aspects of the inflammatory process, such as enhancement of vascular permeability and chemotactic attraction of polymorphonuclear leukocytes (PMNs) and macrophages. In the present study, 19 of the symptomatic cases were associated with purulent exudates, and a positive correlation was found between the presence of purulent exudates and the concentration of endotoxin. Simon et al. (1971) found a statistically significant correlation between the quantity of endotoxin in gingival exudates and the clinical degree of gingival inflammation.

A positive association was found between cases with endodontic signs and symptoms such as spontaneous pain, tenderness to percussion, pain on palpation, swelling and purulent exudates and the concentration of endotoxin, whereas a negative association was found between the endotoxin present in the root canals and asymptomatic teeth.

Horiba et al. (1991) examined the endotoxin content of samples obtained from single root canals of 30 teeth displaying apical periodontitis. Their results showed not only that teeth with clinical symptoms have high levels of endotoxin but also that the detection rates of endotoxin and the mean endotoxin content were higher for teeth with exudation than for teeth with dry root canals, which supports the findings of the present study. On the other hand, Wesselink et al. (1978) investigated the role of endotoxin by using an experimental model simulating the root canal and concluded that the primary toxicity of endotoxin extracted from an LPS-B had no major part in the initiation or maintenance of chronic periapical inflammation. The evaluation was made histologically by screening for signs of the Shwartzman reaction.

The concentrations of endotoxin found in all the cases of this study were higher than those reported in previous studies (Dahlen \& Bergenholtz, 1980; Horiba et al., 1991; Khabbaz et al., 2000, 2001; Schein \& Schilder, 1975). However, it is not possible to compare the numbers of this study to others due to differences in protocols or clinical features investigated. Dahlen \& Bergenholtz (1980) aspirated with a syringe the suspended contents of the root canal and transferred it to $1 \mathrm{ml}$ of sterile $\mathrm{NaCl}$ solution. Khabbaz et al. (2001) analysed cases of irreversible pulpitis; the pulpal tissue was removed with a sterile barbed breach and then transferred to a preweighed pyrogen-free tube. In this study only infected teeth with periapical bone destruction were analysed and five paper points were inserted in the full-length of the root canal for $1 \mathrm{~min}$. The aim was to exhaustively remove the endotoxin content from the infected tooth, consequently a high concentration of endotoxin would be predicted.

Pathogenic factors other than endotoxin may be involved in the inflammatory processes taking place in the periapical area adjacent to the infected root canal. However, the concentration of endotoxin in the root canal of teeth with necrotic pulpal tissue found in this study, and the higher concentration of endotoxin in cases of teeth with spontaneous pain, pain on palpation, tenderness to percussion and purulent exudates, indicates that LPS is a significant biological factor for the development of acute periapical inflammation. Moreover, micro-organisms and their products, like endotoxin, arising from a circumscribed endodontic infection might disseminate systemically, resulting in the initiation or exacerbation of systemic illness or damage at a distant tissue site (Debelian et al., 1995; Murray \& Saunders, 2000). This highlights the need for using, during endodontic treatment, substances that not only act as antimicrobial agents but also have the ability to inactivate bacterial products such as endotoxin.

This study demonstrated unequivocally that endotoxin is present in high concentrations in root canals of infected teeth 
(a)

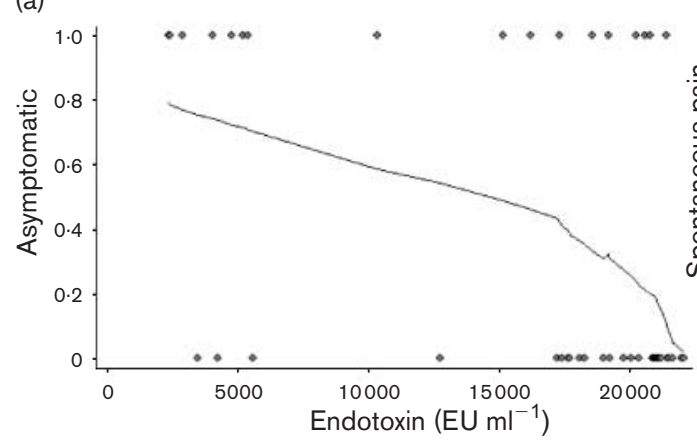

(c)

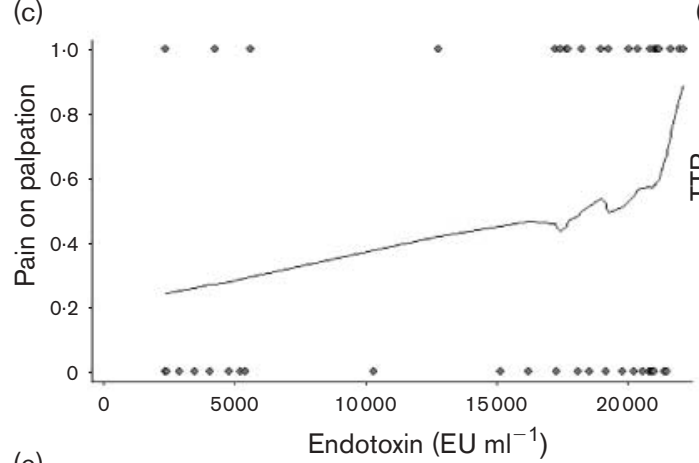

(b)

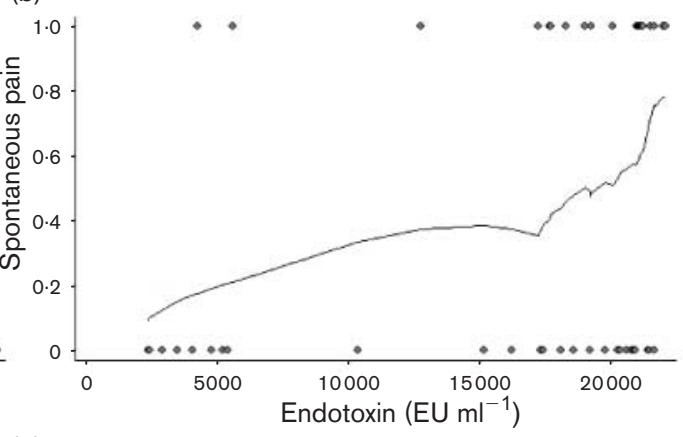

(d) (e)

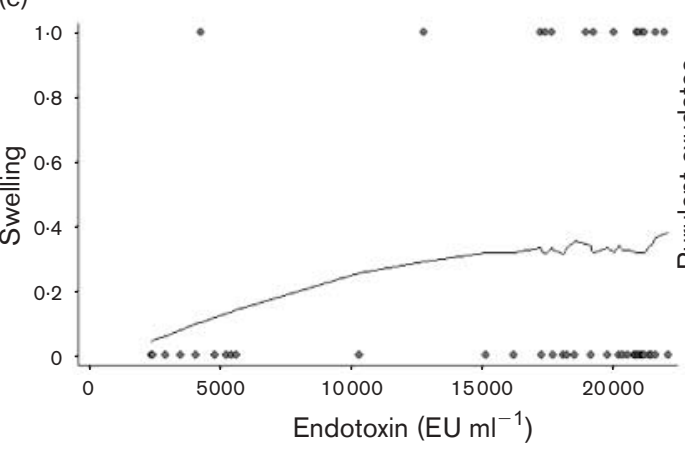

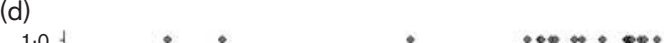

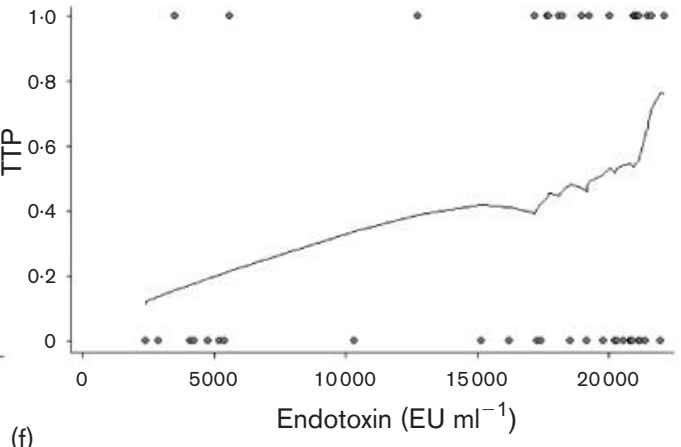

(f)

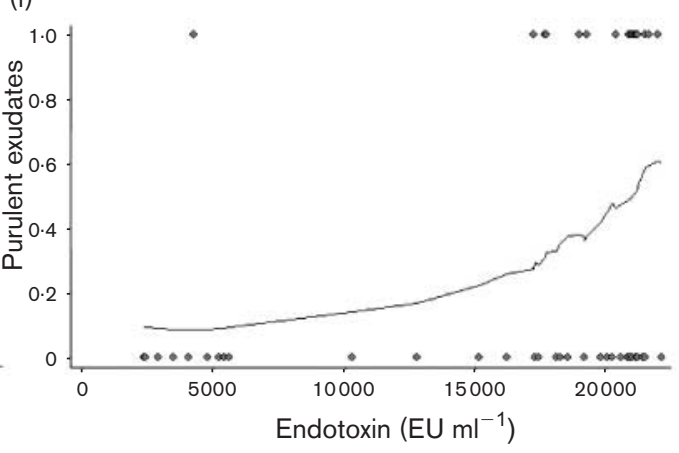

Fig. 3. Locally weighted regression plot of the concentration of endotoxin in relation to endodontic signs and symptoms. The vertical axes indicate (a) occurrence of asymptomatic teeth, (b) spontaneous pain, (c) pain on palpation, (d) tenderness to percussion, (e) swelling and ( $f$ ) purulent exudates. The asymptomatic profile contrasts sharply with those showing symptoms. Bandwidth 0.8 for all graphs.

with endodontic symptoms and that there is a positive correlation between the concentration of endotoxin in the root canal and the presence of endodontic signs and symptoms.

\section{ACKNOWLEDGEMENTS}

We would like to thank Miss Susan Nunn from the Statistics Unit at the Health Protection Agency, Communicable Disease Surveillance Centre, for invaluable statistical help and Mr Adailton dos Santos Lima for technical support. This work was supported by the Brazilian agencies FAPESP (2000/13689-7, 2000/13686-8, 2000/13683-9, 2002/08167-7), CNPq (520277/99-6 \& 304282/2003-0) and CAPES BEX (2801/03-5).

\section{REFERENCES}

Baumgartner, J. C. (1991). Microbiologic and pathologic aspects of endodontics. Curr Opin Dent 1, 737-743.
Dahlen, G. (1980). Immune response in rats against lipopolysaccharides of Fusobacterium nucleatum and Bacteroides oralis administered in the root canal. Scand J Dent Res 88, 122-129.

Dahlen, G. \& Bergenholtz, G. (1980). Endotoxic activity in teeth with necrotic pulps. J Dent Res 59, 1033-1040.

Dahlen, G. \& Hofstad, T. (1977). Endotoxic activities of lipopolysaccharides of microorganisms isolated from an infected root canal in Macaca cynomolgus. Scand J Dent Res 85, 272-278.

Debelian, G. J., Olsen, I. \& Tronstad, L. (1995). Bacteremia in conjunction with endodontic therapy. Endod Dent Traumatol 11, $142-149$.

Elin, R. J. \& Wolff, S. M. (1976). Biology of endotoxin. Annu Rev Med 27, $127-141$.

Friberger, P., Knos, M. \& Mellstam, L. (1982). Endotoxins and their Detection with the Limulus Amebocyte Test, pp. 195-206. New York: A. R. Liss. 
Gomes, B. P. F. A., Drucker, D. B. \& Lilley, J. D. (1994). Associations of specific bacteria with some endodontic signs and symptoms. Int Endod J 27, 291-298.

Gomes, B. P. F. A., Lilley, J. D. \& Drucker, D. B. (1996a). Clinical significance of dental root canal microflora. J Dent 24, 47-55.

Gomes, B. P. F. A., Drucker, D. B. \& Lilley, J. D. (1996b). Association of endodontic symptoms and signs with particular combinations of specific bacteria. Int Endod J 29, 69-75.

Gomes, B. P. F. A., Pinheiro, E. T., Gade-Neto, C. R., Sousa, E. L., Ferraz, C. C., Zaia, A. A., Teixeira, F. B. \& Souza-Filho, F. J. (2004). Microbiological examination of infected dental root canals. Oral Microbiol Immunol 19, 71-76.

Griffee, M. B., Patterson, S. S., Miller, C. H., Kafrawy, A. H. \& Newton, C. W. (1980). The relationship of Bacteroides melaninogenicus to symptoms associated with pulpal necrosis. Oral Surg Oral Med Oral Pathol 50, 457-461.

Haapasalo, M., Ranta, H., Ranta, K. \& Shah, H. (1986). Black-pigmented Bacteroides spp. in human apical periodontitis. Infect Immun 53, 149-153.

Hashioka, K., Yamasaki, M., Nakane, A., Horiba, N. \& Nakamura, H. (1992). The relationship between clinical symptoms and anaerobic bacteria from infected root canals. J Endod 18, 558-561.

Horiba, N., Maekawa, Y., Matsumoto, T. \& Nakamura, H. (1990). A study of the distribution of endotoxin in the dentinal wall of infected root canals. J Endod 16, 331-334.

Horiba, N., Maekawa, Y., Abe, Y., Ito, M., Matsumoto, T. \& Nakamura, H. (1991). Correlations between endotoxin and clinical symptoms or radiolucent areas in infected root canals. Oral Surg Oral Med Oral Pathol 71, 492-495.

Jacinto, R. C., Gomes, B. P. F. A., Ferraz, C. C. R., Zaia, A. A. \& Souza-Filho, F. J. (2003). Microbiological analysis of infected root canals from symptomatic and asymptomatic teeth with periapical periodontitis and the antimicrobial susceptibility of some isolated anaerobic bacteria. Oral Microbiol Immunol 18, 285-292.

Khabbaz, M. G., Anastasiadis, P. L. \& Sykaras, S. N. (2000). Determination of endotoxins in caries: association with pulpal pain. Int Endod J 33, 132-137.

Khabbaz, M. G., Anastasiadis, P. L. \& Sykaras, S. N. (2001).
Determination of endotoxins in the vital pulp of human carious teeth: association with pulpal pain. Oral Surg Oral Med Oral Pathol Oral Radiol Endod 91, 587-93.

Matsushita, K., Tajima, T., Tomita, K., Takada, H., Nagaoka, S. \& Torii, M. (1999). Inflammatory cytokine production and specific antibody responses to lipopolysaccharide from endodontopathic black-pigmented bacteria in patients with multilesional periapical periodontitis. $J$ Endod 25, 795-799.

Morrison, D. C. \& Kline, L. F. (1977). Activation of the classical and properdin pathways of complement by bacterial lipopolysaccharides (LPS). J Immunol 118, 362-368.

Murray, C. A. \& Saunders, W. P. (2000). Root canal treatment and general health: a review of the literature. Int Endod J 33, 1-18.

Pitts, D. L., Williams, B. L. \& Morton, T. H., Jr (1982). Investigation of the role of endotoxin in periapical inflammation. J Endod 8, 10-18.

Rolph, H. J., Lennon, A., Riggio, M. P., Saunders, W. P., MacKenzie, D., Coldero, L. \& Bagg, J. (2001). Molecular identification of microorganisms from endodontic infections. J Clin Microbiol 39, 3282-3289.

Rosengren, C. \& Winblad, B. (1975). Periapical destructions caused by experimental pulpal inoculation of Streptococcus mutans in rats. Oral Surg 39, 479-487.

Schein, B. \& Schilder, H. (1975). Endotoxin content in endodontically involved teeth. J Endod 1, 19-21.

Seltzer, S. \& Farber, P. A. (1994). Microbiologic factors in endodontology. Oral Surg Oral Med Oral Pathol 78, 634-645.

Simon, B. I., Goldman, H. M., Ruben, M. P. \& Baker, E. (1971). The role of endotoxin in periodontal disease. Correlation of the amount of endotoxin in human gingival exudate with the histologic degree of inflammation. J Periodontol 42, 210-216.

Sundqvist, G., Johansson, E. \& Sjogren, U. (1989). Prevalence of blackpigmented bacteroides species in root canal infections. J Endod 15, 13-19.

Wesselink, P. R., Thoden van Velzen, S. K. \& Makkes, P. C. (1978). Release of endotoxin in an experimental model simulating the dental root canal. Oral Surg Oral Med Oral Pathol 45, 789-795.

Yoshida, M., Fukushima, H., Yamamoto, K., Ogawa, K., Toda, T. \& Sagawa, H. (1987). Correlation between clinical symptoms and microorganisms isolated from root canals of teeth with periapical pathosis. J Endod 13, 24-28. 